\title{
Hydrogenated Amorphous Silicon Sensor Deposited on Integrated Circuit for Radiation Detection
}

\author{
M. Despeisse, G. Anelli, P. Jarron, J. Kaplon, D. Moraes, A. Nardulli, F. Powolny, and N. Wyrsch
}

\begin{abstract}
Radiation detectors based on the deposition of a 10 to $30 \mu \mathrm{m}$ thick hydrogenated amorphous silicon $(\mathbf{a}-\mathrm{Si}: \mathrm{H})$ sensor directly on top of integrated circuits have been developed. The performance of this detector technology has been assessed for the first time in the context of particle detectors. Three different circuits were designed in a quarter micron CMOS technology for these studies. The so-called TFA (Thin-Film on ASIC) detectors obtained after deposition of a-Si:H sensors on the developed circuits are presented. High internal electric fields $\left(10^{4}\right.$ to $\left.10^{5} \mathrm{~V} / \mathrm{cm}\right)$ can be built in the a-Si:H sensor and overcome the low mobility of electrons and holes in this amorphous material. However, the deposited sensor's leakage current at such fields turns out to be an important parameter which limits the performance of a TFA detector. Its detailed study is presented as well as the detector's pixel segmentation. Signal induction by generated free carrier motion in the a-Si:H sensor has been characterized using a $660 \mathrm{~nm}$ pulsed laser. Results obtained with a TFA detector based on an ASIC integrating 5 ns peaking time pre-amplifiers are presented. Direct detection of 10 to $50 \mathrm{keV}$ electrons and $5.9 \mathrm{keV} \mathrm{X}$-rays with the detectors are then shown to understand the potential and the limitations of this technology for radiation detection.
\end{abstract}

Index Terms-Amorphous semiconductors, CMOS analog integrated circuits, detectors.

\section{INTRODUCTION}

$\mathbf{R}$ ADIATION detectors based on crystalline silicon are well established and the associated detector technologies are quite mature. Planar silicon sensors are extensively used for track finding and particle analysis in large High-Energy Physics (HEP) experiments and are also widely operated in biomedical or molecular imaging applications [1]. However, the eventual upgrade of the Large Hadron Collider (LHC) at CERN to a Super-LHC with a 10 times higher luminosity will lead to total radiation levels in some regions of tracking systems that conventional silicon detector structures cannot withstand ([2], [3]). Alternative detector solutions with improved speed and spatial resolution will also be required for the future International Linear Collider (ILC) or the eventual compact linear collider (CLIC) [2]. Therefore, in addition to state-of-the-art hybrid silicon pixel detectors, a number of trends and innovations aimed at improving performance are being developed to greater maturity [1]. The characterization and development of existing and

Manuscript received September 5, 2007; revised January 7, 2008.

M. Despeisse, G. Anelli, P. Jarron, J. Kaplon, D. Moraes, and F. Powolny are with the European Organization for Nuclear Research (CERN), CH-1211 Geneva 23, Switzerland (e-mail: matthieu.despeisse@ cern.ch).

A. Nardulli is with ETH Zurich, Institute for Particle Physics (IPP), CH-8093 Zlrich, Switzerland.

N. Wyrsch is with IMT, University of Neuchatel, CH-2000 Neuchatel, Switzerland.

Digital Object Identifier 10.1109/TNS.2008.918519

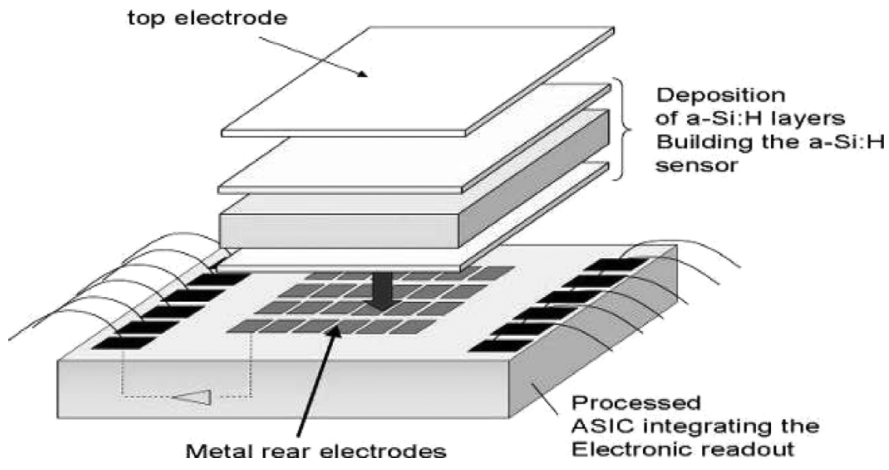

Fig. 1. Schematic representation of a TFA detector. An a-Si:H sensor is deposited directly on top of an ASIC which integrates the electronic readout. Electrodes segmenting the detector are integrated in the ASIC.

emerging detection technologies are of primary interest for future HEP experiments [2] but also for bio-medical imaging applications where alternative solutions to conventional silicon detectors are needed. In this context, an emerging alternative solid state detector technology has been studied. This technology, so-called Thin Film on ASIC (TFA), is based on the deposition of a hydrogenated amorphous silicon (a-Si:H) sensor directly on top of the readout integrated circuit (Fig. 1) ([4], [5]). Its performance has been assessed for the first time in the context of particle detectors.

Hydrogenated amorphous silicon has been studied over the past 30 years and though it is now well understood it still presents controversial issues. a-Si:H is widely industrialized in thin films (sensor thickness below $1 \mu \mathrm{m}$ ) for solar cells and various sensitive imaging devices. Its radiation hardness was studied in the context of testing solar cells for space applications and it was shown that proton, neutron and electron irradiations ([6]-[8]) or photon irradiation (involving the Staebler-Wronski effect [9]) all lead to the creation of metastable deep defects that can be thermally annealed out. Full recovery of proton-induced defects was observed after subsequent room temperature thermal annealing [7] (higher temperature leading to faster recovery), while effective annealing of defects created by room temperature light irradiation was observed for annealing temperature above $120^{\circ} \mathrm{C}$ [9]. The annealing temperature and full recovery time were shown to depend on the irradiation conditions (temperature, type of radiation) and systematic studies still need to be done on this material to conclude on its potential higher radiation hardness compared to crystalline silicon.

a-Si:H has the advantage to be deposited at low cost and on large areas at temperatures below $250^{\circ} \mathrm{C}$, which are compatible with processed integrated circuits. The TFA detector structure (Fig. 1) eliminates the need for bump bonding and enables a 
level of integration comparable to monolithic pixel detectors. Moreover, the detector still presents the advantages of the hybrid pixel approach as the sensing device and the electronic readout can still be designed, optimized and biased separately. In comparison to commercial a-Si:H devices $(<1 \mu \mathrm{m})$, thicker sensors have to be deposited and fully depleted to provide adequate signal to noise ratio for particle detection [5]. The first technological challenge is the deposition of a-Si:H layers with a thickness higher than $30 \mu \mathrm{m}$ and on ASIC. The sensor has to be developed with a minimal defect density and with minimal leakage currents for the high reverse biases needed in order to allow full depletion of the sensor [10]. The voltage $V_{d}$ needed to deplete an a-Si:H sensor of thickness $\mathrm{d}$ (in $\mu \mathrm{m}$ ) can be estimated by considering the mean density of ionized dangling bonds of the material as $\mathrm{N}_{\mathrm{db}}{ }^{*} \sim 6 \times 10^{14} \mathrm{~cm}^{-3}$ and is given by $\mathrm{V}_{\mathrm{d}} \sim 0.48 \times \mathrm{d}_{(\mathrm{in} \mu \mathrm{m})}{ }^{2}[10]$. Sensors with a thickness above $50 \mu \mathrm{m}$ are difficult to grow and would moreover necessitate reverse voltages of more than $1.2 \mathrm{kV}$ for full depletion. Sensors with thicknesses up to $32 \mu \mathrm{m}$ have thus been deposited in our studies.

The developed sensors can be considered to be thick in comparison to standard a-Si:H devices, but they can also be considered thin in comparison to standard silicon detectors (which have thicknesses above $150 \mu \mathrm{m}$ ). Fewer charges are expected to be created by a particle going through a TFA detector than through a standard silicon detector, resulting in the most important limitation and challenge of the TFA technology. Different groups have demonstrated that the signal created by a particle in an a-Si:H sensor deposited on glass is small [11], [12]. Minimum Ionizing Particles (MIP) are expected to create few hundreds of electrons in $20 \mu \mathrm{m}$ thick sensors. The development of this technology requires the design of very low noise amplifiers $\left(<100 \mathrm{e}^{-} \mathrm{rms}\right)$ able to read out the small signal induced by a particle going through the a-Si:H sensor. A pixel detector based on TFA technology appears as an attractive solution but several important challenges have to be overcome for its development.

\section{DeVelopment of TFA Detectors}

The near-monolithic structure of a TFA detector will be presented. The performance of the three integrated circuits developed for these studies will be discussed in detail.

\section{A. Detector Structure}

The a-Si:H sensor is built on top of the ASIC by consecutive depositions of $\mathrm{n}$-doped, intrinsic and $\mathrm{p}$-doped a-Si:H films forming a n-i-p diode. Depositions were done at the Institute of Micro-technology of Neuchâtel (IMT) by Plasma Enhanced Chemical Vapor Deposition at very high frequency of $70 \mathrm{MHz}$ and at $200^{\circ} \mathrm{C}$, using a hydrogen dilution of Silane. A deposition rate of $15.6 \AA / \mathrm{s}$ was obtained [10]. The n-layer and p-layer permit the application of reverse voltages on the i-layer but have high defect density, resulting in important recombination of charge carriers generated in these layers. The doped layers are therefore made very thin $(\sim 30 \mathrm{~nm})$ and the n-i-p diode thickness is practically equivalent to the i-layer thickness.

The a-Si:H sensor bottom contacts (anodes) are defined by metal structures integrated in the ASIC and designed in the topmost metal layer of the technology (Fig. 2). These metal pads

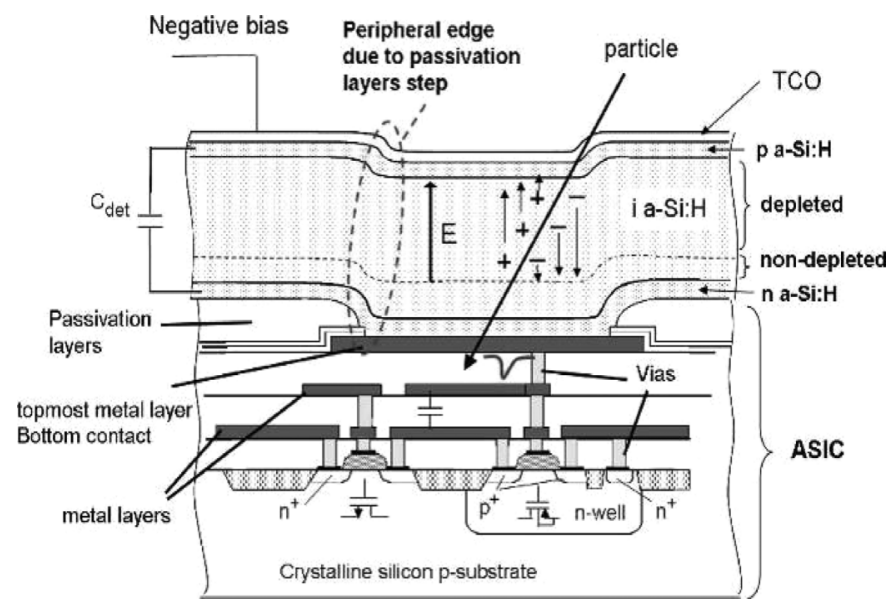

Fig. 2. Schematic cross section of a TFA detector made of n-i-p a-Si:H layers and TCO electrode deposited on top of a CMOS circuit with 3 levels of metal.

define the detector segmentation but are inter-connected through the sensor n-layer. In order to avoid a patterning of this layer, it is specially designed with low conductivity to provide pixel isolation higher than $10 \mathrm{M} \Omega$. A Transparent Conductive Oxide (TCO) is deposited on the sensor p-layer and defines the detector's common top electrode (cathode); this TCO layer is usually made of ITO (Indium Tin Oxide). A schematic cross section of a TFA detector is shown in Fig. 2.

\section{B. Development of Integrated Circuits}

a-Si:H sensors were deposited on 3 ASICs: AFP, Macropad and aSiHtest. The 3 circuits were designed in a quarter micron CMOS technology. They contain different metal pads acting as the detector's electrodes, each pad being connected to an analog electronic readout. The AFP chip contains thirty-two $68 \mu \mathrm{m} \times 94 \mu \mathrm{m}$ pads, each connected to a pre-amplifier with 5 ns peaking time for an Equivalent Noise Charge (ENC) of 400 $\mathrm{e}^{-} \mathrm{rms}$ at $1 \mathrm{pF}$ detector capacitance [13], [14]. The Macropad circuit consists of 48 octagonal pads with $140 \mu \mathrm{m}$ width and $380 \mu \mathrm{m}$ pitch; each pad being connected to an amplifier with a peaking time of $160 \mathrm{~ns}$ and an ENC of $27 \mathrm{e}^{-} \mathrm{rms}$ at $1 \mathrm{pF}$ input capacitance [15].

The aSiHtest circuit is a test chip to characterize detector technologies based on the direct deposition of a sensor on top of an ASIC. A total of 45 individual or grouped metal structures designed in the topmost metal layer are placed in the central area of the chip, defining the active area of the TFA detector (Fig. 3). In CMOS technologies, circuits are protected with $5 \mu \mathrm{m}$ thick passivation layers which cover their surface. Openings in these layers are made to allow external access to the pads made in the topmost metal layer, and in TFA detectors to provide back contacts to the a-Si:H diode. The metal pads and the openings define the detector segmentation. Design rules are such that the openings have to be done only above metal, i.e., inside the pads, so that the passivation layers induce unevenness on the circuit and of the TFA detector surfaces (as shown in Figs. 2-3). However, accepted design rules violations permitted to place in the aSiHtest chip some pads for which the openings are done on top and all around the metal pads (i.e., also on top of the $\mathrm{SiO}_{2}$ 


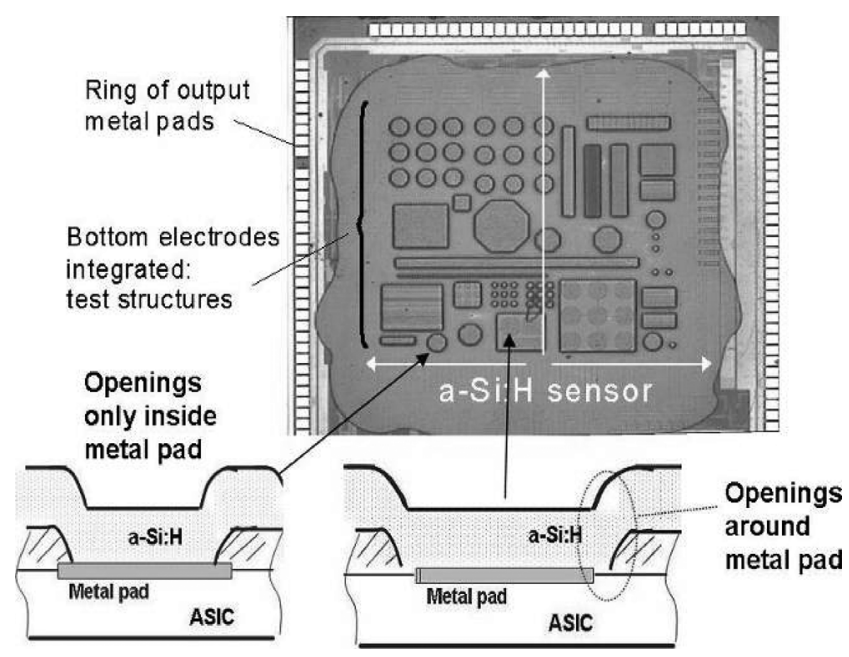

Fig. 3. Picture of a TFA detector based on a $20 \mu \mathrm{m}$ thick n-i-p a-Si:H diode deposited on top of an aSiHtest integrated circuit.

separating metal pads), in order to study the impact of the passivation layers step on detector performance (Fig. 3).

A first group of 21 structures with areas of $18638 \mu \mathrm{m}^{2}$ or $167747 \mu \mathrm{m}^{2}$, with different shapes and with openings in the passivation layers only inside or all around the metal structures are placed in the central area of the chip. Each structure is connected to current mirror stages which provide a selectable current gain of $10^{2}$ to $10^{5}$. The central area of the circuit also contains 24 other metal structures (octagons with areas of 18638 $\mu \mathrm{m}^{2}$ and $2070 \mu \mathrm{m}^{2}$, strips with area of $1864 \mu \mathrm{m}^{2}$ with a pitch varying from $30 \mu \mathrm{m}$ to $10 \mu \mathrm{m})$. Each of these metal structures is connected to one of two implementations of a transimpedance active feedback pre-amplifier [13]. The first implementation (so-called HiSpeed) was optimized to have $5 \mathrm{~ns}$ peaking time and an ENC of $160 \mathrm{e}^{-} \mathrm{rms}$. The second implementation (so-called LoNoise) is a pre-amplifier with $25 \mathrm{~ns}$ peaking time for an ENC of $70 \mathrm{e}^{-} \mathrm{rms}$. Performance of the pre-amplifiers were measured by injecting voltage steps to $100 \mathrm{fF}$ series capacitances integrated in the chip at each pre-amplifier input. Experimental results are shown in Fig. 4.

TFA detectors built on the AFP, Macropad and aSiHtest circuits are respectively presented in [14], [15] and Fig. 3. The characteristics of the developed ASICs are shown in Table I.

\section{EXPERIMENTAL RESULTS}

The 3 developed ASICs have different noise and speed optimizations of the integrated pre-amplifiers and different metallic pads layout (Table I) so that the TFA detectors we developed on these circuits (Table II) permit different characterizations of the technology. The main experimental results will be presented here. The leakage current mechanisms in an a-Si:H sensor and specificities linked to its deposition on a circuit will first be detailed in Section III-A. The signal induction in an a-Si:H sensor was studied by transient current techniques and will be presented in Section III-B. Finally, ionizing radiation detection results will be shown in Section III-C to understand the potential and the limitations of this detection technology. The specific characterizations done on each developed TFA detector are summarized in Table II.

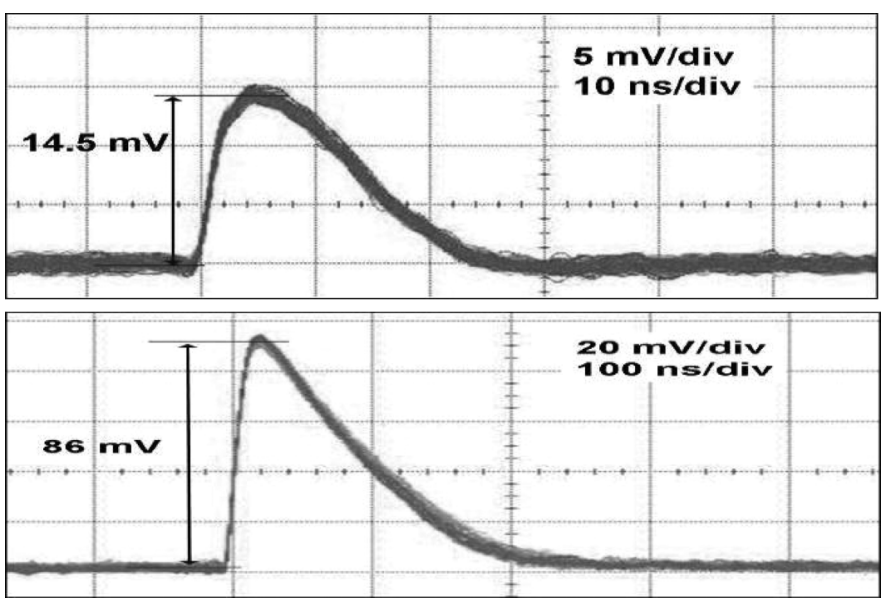

Fig. 4. Response to a $1 \mathrm{fC}$ input charge of active feedback pre-amplifiers integrated in the aSiHtest circuit. A total of 15000 output signals are superposed. Top: Response of the HiSpeed version. Vertical scale is $5 \mathrm{mV} / \mathrm{div}$ and horizontal scale is $10 \mathrm{~ns} /$ div. Bottom: response of the LoNoise version. Vertical scale is 20 $\mathrm{mV} /$ div and horizontal scale is $100 \mathrm{~ns} / \mathrm{div}$.

TABLE I

DESCRIPTION OF THE AFP, MACROPAD AND ASIHTEST ASICS DEVELOPED TO CHARACTERIZE THE POTENTIAL OF TFA TECHNOLOGY FOR RADIATION DETECTION

\begin{tabular}{|c|c|c|c|c|}
\hline \multirow[b]{2}{*}{ ASIC } & \multicolumn{2}{|r|}{ Input pads } & \multicolumn{2}{|c|}{ Pre-amplifier } \\
\hline & $\begin{array}{l}\mathrm{Nu} \\
\mathrm{m}- \\
\text { ber }\end{array}$ & Size / Type & $\begin{array}{l}\text { Peak } \\
\text { time } \\
\text { (ns) }\end{array}$ & $\begin{array}{l}\text { ENC } \\
\left(\mathrm{e}^{-}\right. \\
\mathrm{rms})\end{array}$ \\
\hline AFP & 32 & $\begin{array}{l}68 \mu \mathrm{m} \times 94 \mu \mathrm{m} / \\
20 \mu \mathrm{m} \text { separation }\end{array}$ & 5 & 400 \\
\hline $\begin{array}{c}\text { Macro } \\
\text { pad }\end{array}$ & 48 & $\begin{array}{l}\text { Octagon } 140 \mu \mathrm{m} \\
240 \mu \mathrm{m} \text { separation }\end{array}$ & 160 & 30 \\
\hline \multirow{5}{*}{$\begin{array}{c}\text { aSiH } \\
\text { test }\end{array}$} & \multirow{4}{*}{$\begin{array}{l}7 \\
8\end{array}$} & \multirow{4}{*}{$\begin{array}{l}\text { Octagons: } 18638 \mu \mathrm{m}^{2} \text { or } \\
2070 \mathrm{~m}^{2} \\
6.6 \mu \mathrm{m} \times 282 \mu \mathrm{m} \text { strips, pitch } \\
\text { from } 30 \mu \mathrm{m} \text { to } 10 \mu \mathrm{m}\end{array}$} & \multicolumn{2}{|c|}{ HiSpeed } \\
\hline & & & 5 & 160 \\
\hline & & & \multicolumn{2}{|c|}{ LoNoise } \\
\hline & & & 25 & 70 \\
\hline & 21 & $\begin{array}{c}18638 \mu \mathrm{m}^{2} \text { or } 167747 \mu \mathrm{m}^{2} \\
\text { different shapes, passivation } \\
\text { openings only in or around } \\
\text { metal pad }\end{array}$ & \multicolumn{2}{|c|}{$\begin{array}{l}\text { Current mirrors } \\
\text { gain } 10^{2} \text { to } 10^{5}\end{array}$} \\
\hline
\end{tabular}

\section{A. a-Si:H Sensor Leakage Current}

Minimum leakage currents of the reverse biased a-Si:H sensor are needed to permit the full depletion of the sensor and to minimize the noise of the detector. The leakage current of standard thin $(\leq 1 \mu \mathrm{m}) \mathrm{n}$-i-p a-Si:H sensors was shown to originate from thermal generation and current densities as low as $10^{-12} \mathrm{~A} / \mathrm{cm}^{2}$ can be achieved [16]. However, thicker layers of 10 to $30 \mu \mathrm{m}$ are used in the detectors developed for particle detection. Results obtained on $32 \mu \mathrm{m}$ thick a-Si:H sensors deposited on a glass substrate are presented in [10], showing a low dark current density of $3 \times 10^{-11} \mathrm{~A} / \mathrm{cm}^{2}$ for a reverse bias voltage of $30 \mathrm{~V}$ but high current densities of $10^{-7} \mathrm{~A} / \mathrm{cm}^{2}$ for $320 \mathrm{~V}$. Moreover, a-Si:H sensors deposited on a Macropad and on an AFP chip exhibited leakage currents up to 3 orders of magnitude higher than for similar sensors deposited on a glass substrate ([14], [15]), preventing the full depletion of the sensor. The high reverse voltages needed to 
TABLE II

SUMMARY OF TFA DETECTORS DEVELOPED (CIRCUIT AND THICKNESS OF DEPOSITED A-SI:H SENSOR) AND OF CHARACTERIZATIONS PERFORMED AND PRESENTED IN SECTION III

\begin{tabular}{|c|c|c|}
\hline ASIC & $\begin{array}{l}\text { a-Si:H } \\
\text { sensor }\end{array}$ & Characterizations \\
\hline AFP & $\begin{array}{l}13 \mu \mathrm{m} \\
32 \mu \mathrm{m}\end{array}$ & $\begin{array}{l}\text { - Detection of keV-electrons : [14] } \\
\text { - TFA segmentation - study of Leakage current } \\
\text { issues: Section III A } \\
\text { - Induced signal speed study: [14]-Section III B }\end{array}$ \\
\hline $\begin{array}{c}\text { Macro } \\
\text { pad }\end{array}$ & $15 \mu \mathrm{m}$ & $\begin{array}{l}\text { - Detection of } 5.9 \mathrm{keV} \text { X-rays [15]- Section III } \\
\underline{\mathbf{C}}\end{array}$ \\
\hline$\underset{\text { test }}{\text { aSiH }}$ & $\begin{array}{l}5 \mu \mathrm{m} \\
10 \mu \mathrm{m} \\
20 \mu \mathrm{m}\end{array}$ & $\begin{array}{l}\text { - Leakage current - TFA segmentation: results } \\
\text { on } 20 \mu \mathrm{m} \text { sensors: } \underline{\text { section III A }} \\
\text { - Induced signal speed and depletion studies: } \\
\text { results on } 20 \mu \mathrm{m} \text { sensors: } \underline{\text { section III B }} \\
\text { - tens of } \mathrm{keV} \mathrm{e} \text { detection w. fine strip structures, } \\
\text { results on } 20 \mu \mathrm{m} \text { sensors: } \underline{\text { section III C }}\end{array}$ \\
\hline
\end{tabular}

deplete the "thick" sensors and the deposition on ASIC surface thus strongly increase the sensor's leakage current, enhancing mechanisms negligible in commercial a-Si:H devices.

An electric field enhancement of thermal generation of charges in the a-Si:H sensor (Poole-Frenkel mechanisms [17]) can explain the bias voltage dependence of the leakage current measured on thick a-Si:H sensors. Moreover, some very high localized electric fields in a TFA detector pixel could explain the high currents measured in the TFA detectors. The pixel active area of a detector made of a $32 \mu \mathrm{m}$ thick diode and of an AFP chip has therefore been studied using a Scanning Electron Microscope (SEM) to measure its homogeneity. A $20 \mathrm{keV}$ electron beam is used to scan the pixel and the signal induced on the bottom electrode is amplified by the integrated pre-amplifier and fed back to the SEM operating system. This generated current depends on the depleted thickness, on the internal electric field which modifies the drift velocities of carriers generated and on the weighting field. An image of these internal properties of the sensor is then built using Electron Beam Induced Current (EBIC), with contrast proportional to the amplitude of the induced signal. Standard precise SEM pictures were also acquired to study the surface of the pixel. The SEM and EBIC images obtained on two pixels of the AFP TFA detector are presented in Fig. 5 (260 V is applied to the sensor).

The SEM picture shows a peripheral edge and some fissures at the corners of the pixel caused by the unevenness of the ASIC surface. The EBIC image clearly shows the effect of this particular geometry on the detector electric field. Higher EBIC currents are observed at the edges and corners, indicating higher electric fields at these regions, which might be responsible of the high dark currents measured in TFA detectors.

These additional leakage currents at a pixel periphery and corners suggested by the EBIC measurements were then verified on detectors made on the aSiHtest chip. Dark currents were measured on the different metal structures and tendencies measured on the different samples are illustrated by results obtained on a $20 \mu \mathrm{m}$ thick sample (Fig. 6). The leakage current density of the different structures varies exponentially with the square
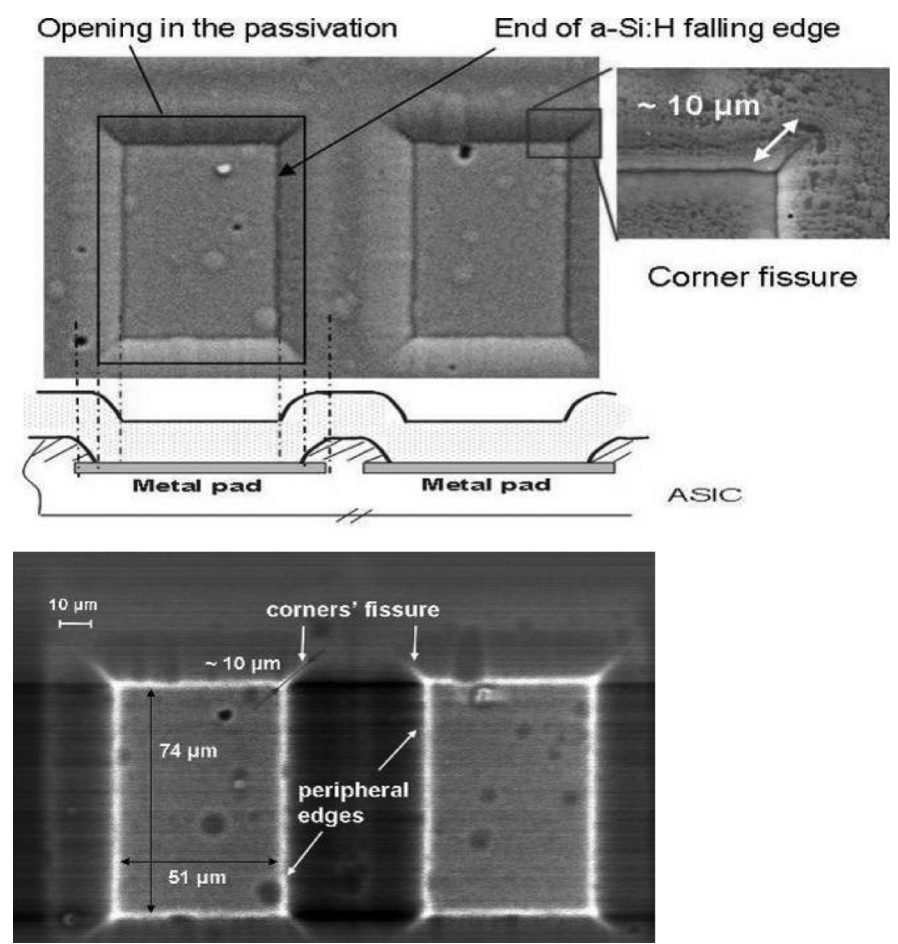

Fig. 5. EBIC study of 2 pixels of a TFA detector made of a $32 \mu \mathrm{m}$ thick a-Si:H sensor on top of an AFP chip. Top: SEM picture showing geometrical effects caused by the passivation layer steps. Center: Schematic cross section of the TFA detector on 2 pixels. Bottom: EBIC image. White zones correspond to higher induced current.

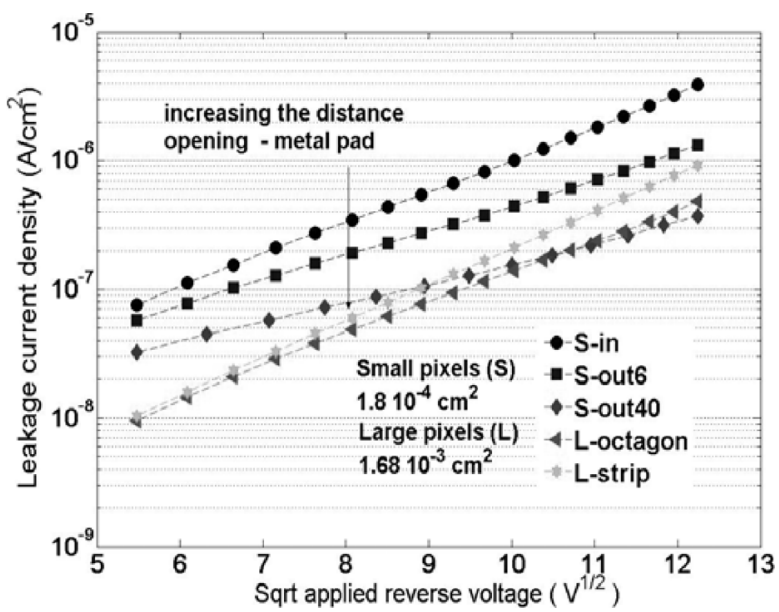

Fig. 6. Leakage current density measured on a $20 \mu \mathrm{m}$ thick sensor on top of an aSiHtest chip. L-octagon is an octagonal structure and L-strip a strip structure both with an opening of the passivation layers inside the metal pad and with an area of $1.68 \times 10^{-3} \mathrm{~cm}^{2}$. S-in, S-out6 and S-out40 are octagons with an area of $1.8 \times 10^{-4} \mathrm{~cm}^{2}$ and with passivation openings respectively inside the metal pad and on top and all around pad at $6 \mu \mathrm{m}$ and at $40 \mu \mathrm{m}$ from the metal pad edges.

root of the applied voltage. Pads with an area of $1.8 \times 10^{-4} \mathrm{~cm}^{2}$ present a current density 6 times lower than pads with a 9 times smaller area, indicating contributions from pixel edges and corners. Measurements were also done on similar metal pads but with openings in the passivation layers either only inside the pad (Fig. 3 left) or on top of the whole pad and all around the pad (Fig. 3 right), at $6 \mu \mathrm{m}$ or $40 \mu \mathrm{m}$ around. Experimental results clearly demonstrate that opening the passivation layers on 


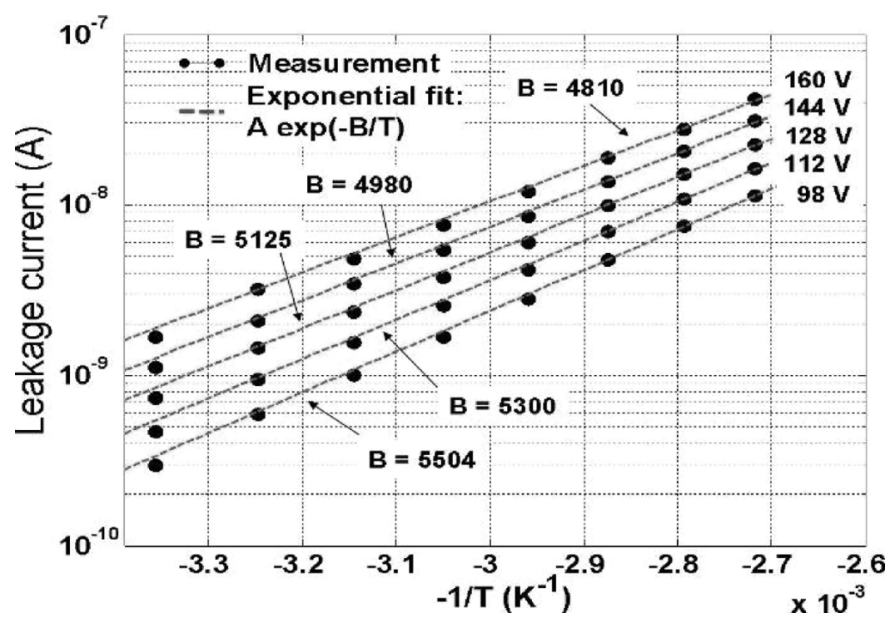

Fig. 7. Evolution of leakage current with temperature measured on a $20 \mu \mathrm{m}$ thick a-Si:H sensor on top of an aSiHtest circuit.

top and all around the metal pad leads to a reduction of the additional currents created at a pixel periphery and edges. When openings are done inside the metal pad, the a-Si:H falling edges are on top of the readout electrode, so that high electric field is built, enhancing the leakage current (EBIC study), while when the opening is made around the pad, the falling edges of the a-Si:H layers are not anymore on top of the metal pad edges but around the pad (as shown in Fig. 3 right). As the edges are moved away from the metal pad, the electric field is reduced in these edges, resulting in a lower thermal generation of charges. The weighting field for charges generated in the edges and the conductance to the metal pad are also reduced, explaining also the reduction of the high additional currents.

The mechanisms responsible for the field enhancement of a TFA detector dark current were also studied by measuring the current's evolution with temperature (Fig. 7). A current induced by thermal generation enhanced by electric field $\mathrm{E}$ through Poole-Frenkel mechanisms can be calculated as [17]:

$$
\begin{aligned}
\mathrm{I} & =\mathrm{I}_{0} \exp \left(\frac{-\mathrm{E}_{\mathrm{A}}}{\mathrm{kT}}\right) ; \\
\mathrm{E}_{\mathrm{A}} & =\frac{\mathrm{E}_{\mathrm{G}}}{2}-\gamma \sqrt{\mathrm{E}} .
\end{aligned}
$$

$\mathrm{E}_{\mathrm{G}}$ is the material bandgap, $\mathrm{E}_{\mathrm{A}}$ is the activation energy, i.e., the energy needed to activate ionisable defects, $\gamma$ is the PooleFrenkel constant, $\mathrm{I}_{0}$ the maximum current (for $\mathrm{E}_{\mathrm{A}}=0$ ), $\mathrm{T}$ the temperature and $\mathrm{k}$ the Boltzmann constant. Experimental data presented in Fig. 7 is well fitted by (1) and (2), showing that the leakage current in the TFA detector can be attributed to Poole-Frenkel reduction of the activation energy, enhancing the thermal generation of charges. The activation energy was extracted for different structures and it was shown to decrease for increasing applied reverse voltages. Values equal to $0.49 \mathrm{eV}$ and $0.42 \mathrm{eV}$ were measured for $80 \mathrm{~V}$ and $160 \mathrm{~V}$ reverse biases respectively, while it is expected to be at about mid-gap, i.e., 0.9 eV, for no applied biases [17].

Experimental results obtained on aSiHtest TFA detectors demonstrate that the a-Si:H sensor leakage current is mainly caused by field enhanced thermal generation and that very high leakage currents are clearly created at a pixel periphery and edges where the electric field gets much higher than in the center of the pixel. Most of the current is also expected to originate from the $\mathrm{p}-\mathrm{i}$ interface which exhibits a higher defect density and the higher electric fields. Global reduction of a TFA detector leakage current can be achieved by opening the passivation layers on top and all around the pixels and by specific optimization of the p-layer ([10], [17]).

\section{B. Characterization of Signal Induction Using Transient Current Technique}

Radiation incident on an a-Si:H sensor generates free charge carriers, either all along its track (charged particle interaction) or more locally (photon interaction involving secondary particles). The free charges which are created in a region of the sensor where an electric field is established move along the field lines and their motion induce a current on the detector electrodes [18]. The variation in time of the induced current was studied on TFA detectors by measuring their response to $660 \mathrm{~nm}$ pulsed laser light. The $660 \mathrm{~nm}$ photons have a mean free path of $1 \mu \mathrm{m}$ in a-Si:H so that when this light pulse impinges on the top electrode, electron-hole pairs are only generated close to the $\mathrm{p}-\mathrm{i}$ interface of the sensor. The application of a reverse voltage on the $n-i-p$ structure gives rise to a stable electric field in the sensor. The electric field has its maximum at the $\mathrm{p}-\mathrm{i}$ interface and then extends into the intrinsic layer where it can be considered in a first approximation as linearly decaying until either it becomes zero at the end of the depleted region (for an under-depleted sensor) or reaches the $\mathrm{n}$ layer (with a non-zero value) for an over-depleted sensor. Electrons generated by the $660 \mathrm{~nm}$ photons drift down through the whole depleted thickness and generated holes drift on a short distance up to the p-layer. The signal induced on the bottom electrode integrated in the ASIC is thus here mainly determined by the electron motion. The moving charges induce a current on the reference electrode which amplitude at time $t$ is proportional to the total drifting charges, to their velocity and to the electrostatic coupling between the moving charges and the reference electrode (weighting field) [18]. Tests were first performed on the $32 \mu \mathrm{m}$ thick sensor deposited on an AFP circuit. Pre-amplifier output signals measured for $3 \mathrm{~ns}$ FWHM laser pulses are presented in Fig. 8. High leakage currents prevented the application of reverse biases above $280 \mathrm{~V}$, so that no full depletion of the a-Si:H sensor could be obtained (a minimum voltage of $460 \mathrm{~V}$ is expected for complete depletion of a $32 \mu \mathrm{m}$ thick sensor).

The signal rise corresponds to the pre-amplifier peaking time ( $\sim 5 \mathrm{~ns})$ and approximately to the time of generation of charges by the laser pulse. The peak thus corresponds to the signal induced by the electron packet moving in the high field region close to the $\mathrm{p}-\mathrm{i}$ interface. The packet drifts under decaying electric field so that the induced signal then decreases. By increasing the detector reverse voltage, both the electric field and the drift velocity of the electrons are increased so that the output signal amplitude is increased (Fig. 8). However, the drift distance (given by the width of the depleted region) is also increased and a globally constant signal decay time of about $20 \mathrm{~ns}$ is measured for all applied biases (Fig. 8). Long tails are 


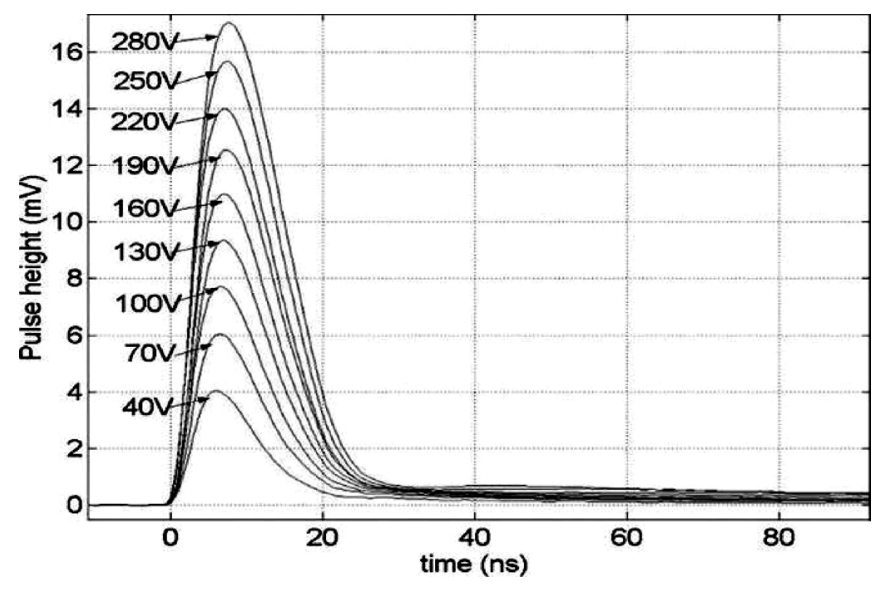

Fig. 8. Response of a $32 \mu \mathrm{m}$ thick a-Si:H sensor on top of an AFP circuit to a $3 \mathrm{~ns}$ FWHM $660 \mathrm{~nm}$ laser pulse for different detector biases. Fast decay of the output pulses correspond to the electron drift.

observed in the output signals, varying with the applied bias and could be caused by holes motion, by electrons drifting in low electric fields or by trapping-detrapping of the moving charges in deep defects.

A simple model of the current induced by the electron motion was developed under the assumption of a linearly decaying electric field. Simplified expressions for the electric field, the depleted thickness $\mathrm{d}$ and the electron motion $\mathrm{x}(\mathrm{t})$ are presented in [4], [5]. Electron transport is non-dispersive in a-Si:H at room temperature and trapping only occurs in shallow localized states, resulting in a globally constant release time and to a constant drift mobility. Electrons created at the p-i interface have therefore a motion along the whole depleted thickness $\mathrm{d}$ defined by:

$$
\begin{aligned}
\mathrm{x}(\mathrm{t}) & =\mathrm{d} \cdot\left(1-\exp \left(\frac{-\mathrm{t}}{\tau_{\mathrm{c}}}\right)\right) ; \\
\tau_{\mathrm{c}} & =\frac{\varepsilon_{0} \cdot \varepsilon_{\mathrm{aSi}}}{\left(\mathrm{q} \cdot \mu_{\mathrm{d}} \cdot \mathrm{N}_{\mathrm{db}}{ }^{*}\right)} .
\end{aligned}
$$

The time constant $\tau_{\mathrm{c}}$ of the electron induced signal is inversely proportional to the drift mobility and to the density of ionized dangling bonds (which defines the electric field). The current created by the electrons drifting in the decaying electric field can thus be expressed as:

$$
I(t)=\left\{\begin{array}{ll}
\frac{-q \cdot N}{d_{t o t}} \frac{d \cdot A \cdot t}{\tau_{c} \cdot t_{1}} & \text { for } \mathrm{t}<t_{1} \\
\frac{-q \cdot N}{d_{\text {tot }}} \frac{\mathrm{d} \cdot \mathrm{A}}{\tau_{c}} \exp \left(-\frac{\left(t-t_{1}\right)}{\tau_{c}}\right) & \text { for } \mathrm{t}>\mathrm{t}_{1}
\end{array} .\right.
$$

The factor $\mathrm{A}$ is equal to $2 \tau_{\mathrm{c}} /\left(2 \tau_{\mathrm{c}}+\mathrm{t}_{1}\right)$ where $\mathrm{t}_{1}$ is the duration of the laser pulse. $d_{\text {tot }}$ is the sensor thickness and $q \cdot N$ is the total charge moving. The sensor weighting field for infinite plate electrode approximation is $1 / d_{\text {tot }}$.

Simulations of the AFP pre-amplifier output response to input currents defined by (5) have been performed with HSPICE. Current signals rising in approximately $3 \mathrm{~ns}$ (corresponding to the laser pulse width) and then decaying as defined by (5) permit a very good fitting between the simulated and measured AFP
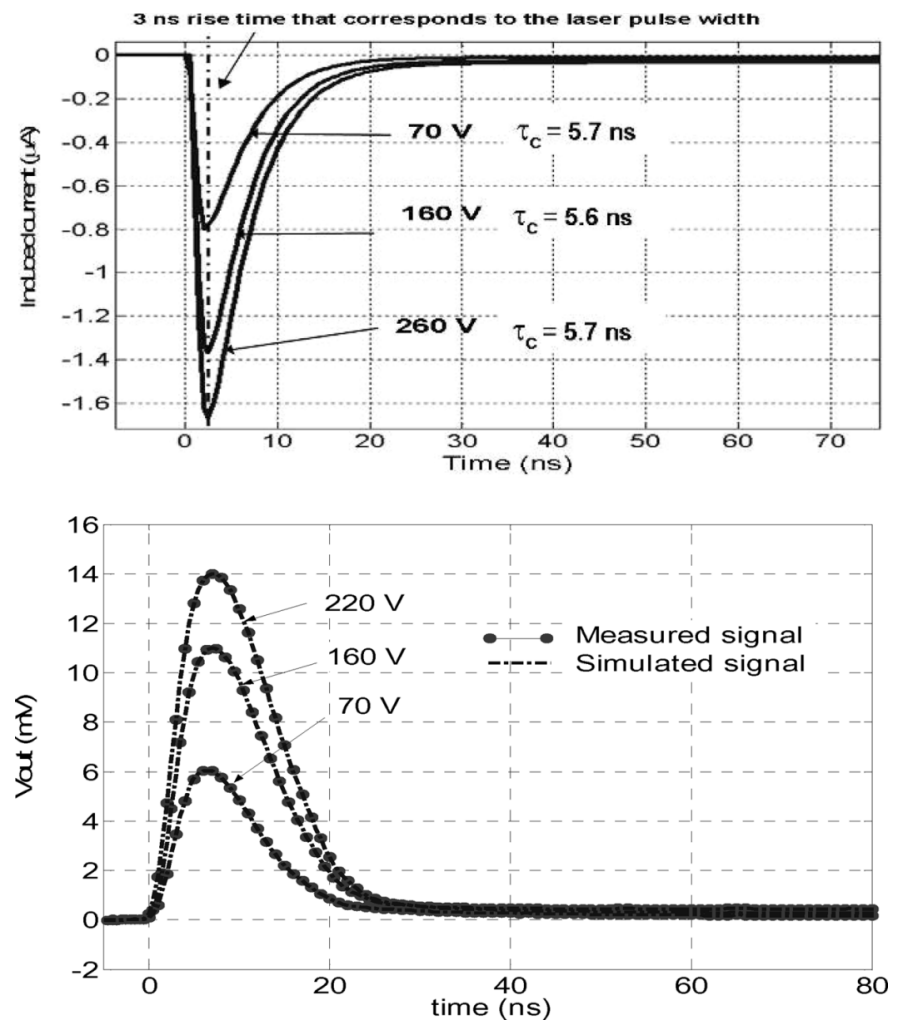

Fig. 9. Top: Calculated currents created by electrons generated at the $\mathrm{p}-\mathrm{i}$ interface during $3 \mathrm{~ns}$ and then drifting in the decreasing electric field. Bottom: simulation of the AFP output response to currents presented on Top. A good fitting of the fast decay component is observed with measured signals.

output response (Fig. 9). The parameter $\tau_{\mathrm{c}}$ was extracted from the simulations and the value obtained was $\tau_{\mathrm{c}}=5.6 \mathrm{~ns}$ for all biases. The product $\mu_{\mathrm{d}} \cdot \mathrm{N}_{\mathrm{db}}{ }^{*}$ was also extracted and is equal to $1.2 \times 10^{15} \mathrm{~cm}^{-1} \mathrm{~V}^{-1} \mathrm{~s}^{-1}$. A density of dangling bonds of about $2 \times 10^{15} \mathrm{~cm}^{-3}$ is expected on our samples ([10]), and by considering an average proportion of $30 \%$ to be ionized, we can estimate to have approximately $\mathrm{N}_{\mathrm{db}}{ }^{*} \sim 6 \times 10^{14} \mathrm{~cm}^{-3}$ [10]. From this expected value, the electron drift mobility was extracted and $\mu_{\mathrm{d}} \sim 2 \mathrm{~cm}^{2} \mathrm{~V}^{-1} \mathrm{~s}^{-1}$, which is in agreement with typical values of 1 to $5 \mathrm{~cm}^{2} \mathrm{~V}^{-1} \mathrm{~s}^{-1}$ in a-Si:H at room temperature. The high electric field inside the a-Si:H sensor $\left(>10^{4} \mathrm{~V} / \mathrm{cm}\right)$ leads to a reasonable drift velocity of the electrons and the induced current lasts for less than $18 \mathrm{~ns}$, even though they have a low mobility.

Similar pulsed laser measurements were performed on a TFA detector made of a $20 \mu \mathrm{m}$ thick a-Si:H sensor deposited on an aSiHtest circuit. Signals measured on pixels connected to the HiSpeed pre-amplifier (Table I) and for biases up to $200 \mathrm{~V}$ are presented in Fig. 10.

A similar fast decay time of less than 20 ns was observed. Simulations of the readout response to input currents defined by (5) were also carried out to fit experimental data, and the time constant $\tau_{\mathrm{c}}$ was estimated, confirming results obtained on the AFP TFA detector. For biases up to $180 \mathrm{~V}$ (voltage at which the sensor is expected to be fully depleted), a $\tau_{\mathrm{c}}$ of approximately $6 \mathrm{~ns}$ was extracted. However, at $200 \mathrm{~V}$, the signal gets faster (Fig. 10) and $\tau_{\mathrm{c}} \sim 5.2 \mathrm{~ns}$. This is an indication of a full depletion of the sensor around $180 \mathrm{~V}$ so that an increase of bias voltage still increases the velocity of the drifting electrons, but does not 


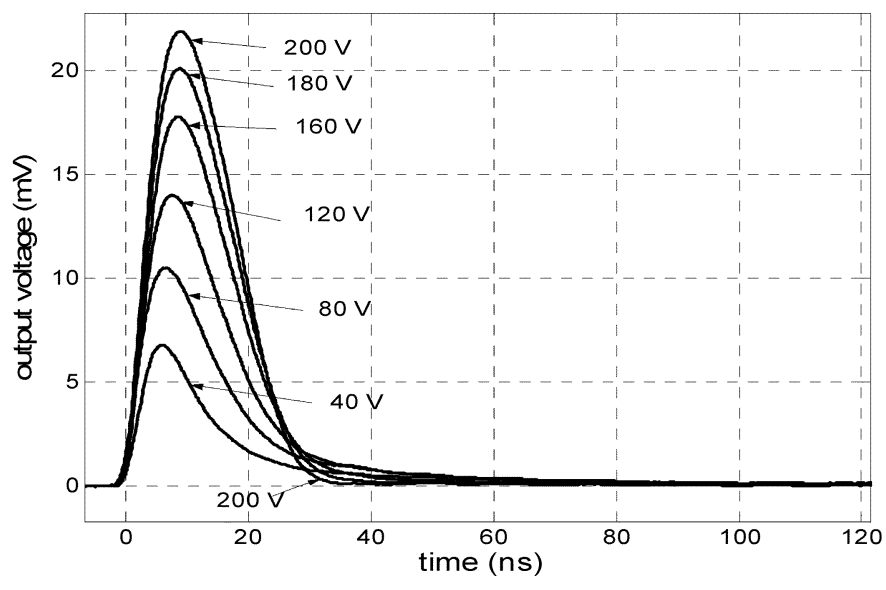

Fig. 10. Response of a $20 \mu \mathrm{m}$ thick a-Si:H sensor on top of an aSiHtest circuit to a $3 \mathrm{~ns}$ FWHM $660 \mathrm{~nm}$ laser pulse for different detector biases. Measurements were performed on a pixel connected to the 1 st version of implemented preamplifier (with 5 ns peaking time).

vary the drift length, resulting in a globally faster induced current. Moreover, the long signal tails observed for low biases end up vanishing at $200 \mathrm{~V}$, while long signal tails (hundreds of ns) were observed at $200 \mathrm{~V}$ on a $32 \mu \mathrm{m}$ thick sensor (Fig. 8). This strongly suggests that the tails measured in under-depleted sensors are for the most caused by electrons moving at the vicinity of the non-depleted region, where very low electric fields are present, and that deep-trapping effects at room temperature are negligible in comparison to this effect. At $200 \mathrm{~V}$ on a $20 \mu \mathrm{m}$ thick sensor, high electric field is expected in the whole sensor, so that no long tail is observed. These results are consistent with the assumption of an electric field linearly decaying but with an exponential behavior in low potential regions ([19]).

Full depletion of the sensor, or more precisely high electric field establishment in the whole sensor, is needed for an optimized signal. We propose to use the same $660 \mathrm{~nm}$ pulsed laser technique to study this condition. Most of the charge read out depends on the integration of the current induced by electron motion. The total induced charge Qe can be calculated by integrating (5) and we can assume that $95 \%$ of this charge will be induced after a time $\mathrm{t}=3 \times \tau_{\mathrm{c}}$. The charge depends on the total number of moving electrons and on the ratio of the depleted thickness over the sensor thickness, as shown below.

$$
\begin{aligned}
\mathrm{Qe} & =\frac{\mathrm{q} \cdot \mathrm{N} \cdot \mathrm{d}}{\mathrm{d}_{\mathrm{tot}}} ; \\
\mathrm{d} & =\sqrt{\frac{2 \cdot \varepsilon_{0} \cdot \varepsilon_{\text {asi }}}{\left(\mathrm{q} \cdot \mathrm{N}_{\mathrm{db}}{ }^{*}\right)}} \cdot \sqrt{\mathrm{V}} .
\end{aligned}
$$

Tests were carried out on a $20 \mu \mathrm{m}$ thick sensor deposited on an aSiHtest chip. Structures connected to LoNoise pre-amplifiers (see Table I) have been characterized. The readout electronic peaking time of $25 \mathrm{~ns}$ is higher than $3 \times \tau_{\mathrm{c}}$ so that the measured output pulse maximum corresponds to the charge Qe read out. Evolution of Qe for varying detector biases is presented in Fig. 11. The charge Qe increases with the square root of the voltage, which is consistent with (6) and (7). The slope depends on $\mathrm{qN}$ and consequently increases for increasing laser

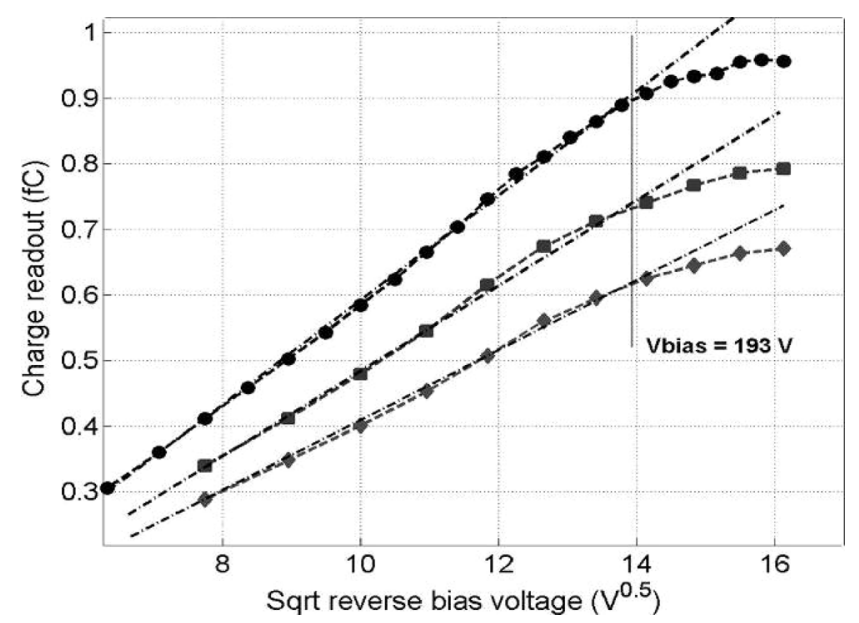

Fig. 11. Charge induced by $3 \mathrm{~ns} 660 \mathrm{~nm}$ laser pulse as a function of the reverse voltage applied on the a-Si:H sensor. Results obtained on a $20 \mu \mathrm{m}$ thick sensor deposited on an aSiHtest chip for 3 different laser amplitudes are shown.

amplitudes. Saturation starts to occur for a reverse voltage of $\sim 193 \mathrm{~V}$, which corresponds to the depletion voltage, as the created charge saturates for $d=d_{\text {tot }}$. This method is successful in determining experimentally the a-Si:H sensor depletion voltage. The mean density of ionized dangling bonds can then be extracted from this measurement and we find $\mathrm{N}_{\mathrm{db}}{ }^{*} \sim 6.4 \times$ $10^{14} \mathrm{~cm}^{-3}$, in agreement with the expected value of $\mathrm{N}_{\mathrm{db}}{ }^{*} \sim$ $6 \times 10^{14} \mathrm{~cm}^{-3}$.

These tests also show that the high-resistivity of the non-depleted region (very low-field region) leads to a reduction of the induced signal, as the charge induced by electrons created at the p-i interface varies as $d / d_{\text {tot }}((6)$ and Fig. 11). A partial depletion of an a-Si:H sensor has thus a double detrimental effect on the induced signal as it not only limits the active volume but also reduces the total charge induced. This strongly confirms the need to lower TFA detectors' leakage current in order to work with fully depleted a-Si:H sensors.

\section{Radiation Response Characterization}

Direct detection of charged particles and of soft X-rays were observed on the developed TFA detectors. The feasibility of detecting charged particles was first demonstrated on a $32 \mu \mathrm{m}$ thick sensor deposited on an AFP circuit. Electrons with energies from $15 \mathrm{keV}$ to $50 \mathrm{keV}$ were detected with a $5 \mathrm{~ns}$ shaping time [4]. Measurements were then done with a $\mathrm{Fe}^{55}$ source on a $15 \mu \mathrm{m}$ thick sensor deposited on a Macropad circuit. Peak amplitudes of the signals created by the $5.9 \mathrm{keV} \mathrm{X}$-rays emitted by the source onto the detector were recorded. A maximum reverse bias of $145 \mathrm{~V}$ could be applied to the sensor (a full depletion is expected for a $100 \mathrm{~V}$ bias) and the spectrum obtained is shown in Fig. 12. A broad peak is observed with a peak charge at about $650 \mathrm{e}^{-}$. The electronic readout has a noise of about $30 \mathrm{e}^{-}$rms but signals were readout in self-trigger mode on a $1.5 \times 10^{-8} \mathrm{~m}^{2}$ pixel area. The source activity is of $10 \mathrm{MBq}$ and the emitting area of few square centimeters. The signal rate on the pixel is thus very low so that in the observed peak the end of the noise Gaussian is still visible. The total induced charge is equivalent to the sum of the charge induced by the motion of generated electrons and by the motion of generated holes. Fast 


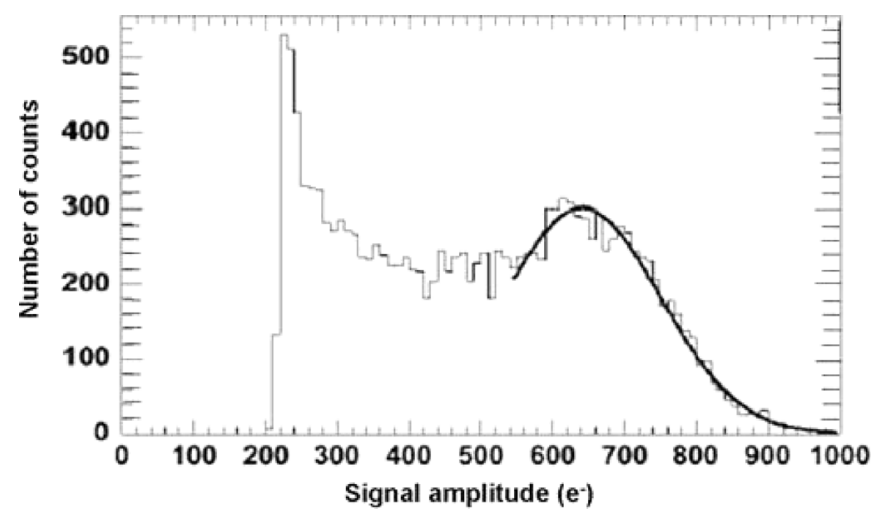

Fig. 12. Spectrum of $5.9 \mathrm{keV} \mathrm{X-rays} \mathrm{from} \mathrm{Fe}^{55}$ source obtained on a $15 \mu \mathrm{m}$ thick a-Si:H sensor deposited on a MACROPAD circuit, for a reverse bias of $145 \mathrm{~V}$ and a threshold of $200 \mathrm{e}^{-}$.

construction of the current induced by the electrons' drift has been shown $(<20 \mathrm{~ns})$, but low and field dependent signal induced by holes was also observed [20]. Hole mobility is less than $1 \%$ of electron mobility and holes have a dispersive transport in a-Si:H [20]. For TFA detectors using pre-amplifiers with a shaping time of few tens of ns, the charge induced by the electrons is expected to be complete while only a small part of the charge induced by the generated holes is integrated because of the slow transport. Depending on the location of creation of the holes and on applied bias, their drift time vary from few tens of ns to few $\mu$ s.

The total charge induced on the detector electrodes by a particle or a photon going through the a-Si:H sensor will thus depend on the location of creation of the electron-hole pairs. The signal induced on the electrodes integrated in the ASIC by pairs created close to the p-i interface will be built principally by the electron drift and is therefore expected to be complete. As pairs are created further into the depleted region, lower signal is created from electron drift and higher contribution from holes is expected. However, as only part of the signal from holes is readout, the induced signal gets lower as the electron-hole pairs are created deeper in the sensor (closer to the readout electrode). A mean energy of creation of electron-hole pairs of 4.8 to $6 \mathrm{eV}$ ([5]) is considered. X-rays interacting close to the $\mathrm{p}-\mathrm{i}$ interface induce a maximum signal. A total maximum charge of 980 to $1230 \mathrm{e}^{-}$is expected, while a maximum signal of about 1000 $\mathrm{e}^{-}$is read out (Fig. 12). X-rays creating pairs further into the sensor will induce a lower signal because of a lower collection of signal from holes, thus the peak has lower amplitude at about $650 \mathrm{e}^{-}$.

Finally, radiation detection experiments were carried out on TFA detectors made of $20 \mu \mathrm{m}$ thick sensors deposited on aSiHtest integrated circuits. Measurements were performed with low energy electrons ( 10 to $50 \mathrm{keV}$ ) on $6.6 \mu \mathrm{m} \times 300 \mu \mathrm{m}$ strip structures with a pitch from $10 \mu \mathrm{m}$ to $20 \mu \mathrm{m}$, each connected to an integrated pre-amplifier with $70 \mathrm{e}^{-} \mathrm{rms}$ noise (Tables I and II). Outputs of 3 neighboring strips were directly read out on an oscilloscope and trigger was performed on the central strip S2 (Fig. 13). Signals created by the 10 to $50 \mathrm{keV}$ electrons interacting in the sensor above the strip S2 are therefore seen, and signals induced at the same time on strip S1 and S3, which are

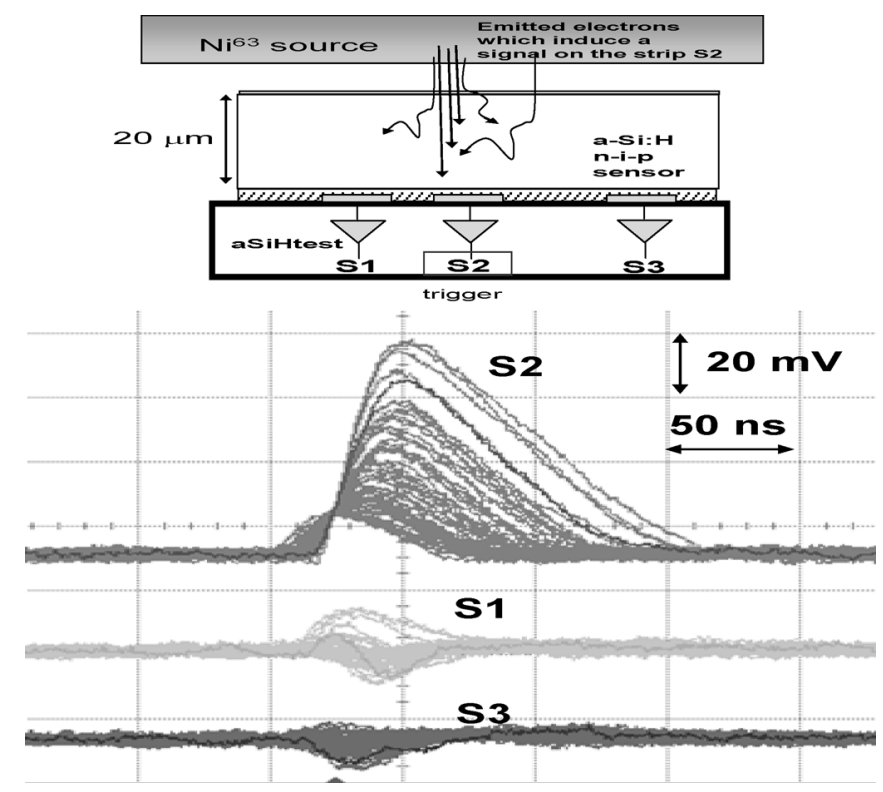

Fig. 13. Signals created by 10 to $50 \mathrm{keV}$ electrons on a $20 \mu \mathrm{m}$ thick a-Si:H sensor deposited on aSiHtest circuit. The circuit is reverse biased with $240 \mathrm{~V}$. Top: readout is done on 3 neighboring strips, S1, S2 and S3, trigger on S2 Bottom: $\sim 100$ superimposed signals created by electrons from the source.

respectively at $4 \mu \mathrm{m}$ and $13.4 \mu \mathrm{m}$ from $\mathrm{S} 2$, are also recorded. The source emits electrons with an energy distribution up to 50 $\mathrm{keV}$, so that the induced signal amplitude shows an exponential distribution following the electron energy distribution (Fig. 13, S2 output, charges of few thousands electrons).

Most of the electrons which induce a signal on the strip S2 do not induce a significant signal on the neighboring strips. The measured transient signals with a low undershoot followed by a low overshoot correspond exactly to the pre-amplifiers' cross-talk and not to induced current (as the observed signals are similar to those observed when injecting an electrical test signal on strip S2). Few signals seen on S2 induce a low signal on $\mathrm{S} 1$, but these events are rare $(<20 \%)$ and can be explained by the tortuous path of few $\mathrm{keV}$ electrons in matter and by events close to the strip boundary (so that some electrons have a track above several strips). Low charge sharing can therefore be considered on very thin strip structures on the developed TFA detectors. The effects of the low diffusion coefficient of about $10^{-2}$ to $10^{-3} \mathrm{~cm}^{2} / \mathrm{s}$ for electrons and about $10^{-4} \mathrm{~cm}^{2} / \mathrm{s}$ for holes [21] are negligible because of the high applied electric field and the short drift times of less than $20 \mathrm{~ns}$ for electrons. Lateral diffusion of an electron packet will be small (less than $1 \mu \mathrm{m} \mathrm{rms}$ for a charge packet created at the p-i interface in a $20 \mu \mathrm{m}$ thick sensor) so that even on the thin strip structure developed low charge sharing between neighboring strips is seen, not reducing the recorded charge. These results show an interesting potential of the TFA technology for the detection of low energy electrons and for the construction of a high spatial resolution detector.

However, no clear proof of Minimum Ionizing Particle (MIP) detection could be obtained up to now on the different developed TFA detectors. MIP detection could be achieved by an increase of the sensor thickness. Lower leakage currents of the a-Si:H sensor can be obtained with the different segmentation techniques we presented in this paper, so that 
$40 \mu \mathrm{m}$ thick sensors could be deposited and fully depleted. A $40 \mu \mathrm{m}$ thick sensor fully depleted could lead to an estimated charge of about 1400 electrons for a MIP, which could be read out by the electronic circuits integrated in the Macropad and aSiHtest circuits. The design of very small pixels (below $100 \mu \mathrm{m}^{2}$ ) could also permit to increase the signal following the small pixel effect [22]. For pixels with dimensions lower than the sensor thickness, the weighting field for the electrode integrated in the ASIC is not anymore constant in the sensor but gets concentrated close to the electrode. Charges drifting in the vicinity of this electrode will therefore determine the induced signal, and in the used n-i-p configuration electrons drift down to the electrode. The induced signal will thus be determined for the most by the motion of electrons and less by the holes, maximizing the charge induced during the integration time of the pre-amplifier.

\section{CONCLUSION}

Thin Film on ASIC detectors were successfully developed by depositing n-i-p a-Si:H sensors with thicknesses ranging from $10 \mu \mathrm{m}$ to $32 \mu \mathrm{m}$ onto 3 different integrated circuits. Preliminary tests performed on a $32 \mu \mathrm{m}$ thick sensor deposited on the AFP circuit (which integrates pre-amplifiers with 5 ns peaking time and $400 \mathrm{e}^{-}$rms noise) demonstrated the possibility to detect ionizing particles with TFA detectors. However signal to noise ratio was shown to be too low for the detection of low ionizing particles with this detector. Moreover, these tests revealed the presence of very high leakage currents due both to the high reverse biases needed to deplete the thick sensor and to the direct deposition on ASIC, preventing the full depletion of the sensor and limiting the detector performance. Measurements performed on TFA detectors based on the aSiHtest circuit demonstrated that the high leakage currents originate from field enhanced thermal generation of charges by Poole-Frenkel mechanisms and that high additional currents are created at every pixel edges and corners of a TFA detector. These additional currents are due to high electric fields at the pixel's periphery created by the unevenness of the ASIC surface. We have shown that opening the passivation layers on top and all around metal pads on the ASIC permits an efficient reduction of this effect, allowing an efficient depletion of the deposited sensor.

Signal induction and speed in a TFA detector were studied using a transient current technique and experimental results show that the signal induced by the electron drift is complete in a time of less than $18 \mathrm{~ns}$. Hole's mobility is less than $1 \%$ of the electron's mobility so that the induced charge read out by the 5 to $150 \mathrm{~ns}$ pre-amplifiers used in our tests is for the most determined by the electron motion. Experimental results show that the non full depletion of the sensor not only reduces the interacting active volume but also reduces the induced charge on the reference electrode integrated in the ASIC. Optimized detection efficiency is therefore clearly achieved with fully depleted sensors. We propose an experimental method based on the use of $660 \mathrm{~nm}$ laser pulses to estimate the depletion condition of the deposited sensor, and we measured a depletion voltage of approximately $190 \mathrm{~V}$ for a $20 \mu \mathrm{m}$ thick sample.
The a-Si:H sensor is thin in comparison to standard crystalline silicon detectors. Moreover a-Si:H presents a higher mean energy of electron-hole pair creation than crystalline silicon $(4.8$ to $6 \mathrm{eV})$, and even if full depletion of the sensor is achieved, maximizing the induced signal, only a part of the signal induced by the holes motion is integrated. These different properties of the technology explain that low signals are created by ionizing particles, giving rise to the most important limitation of the TFA technology. No clear proof of Minimum Ionizing Particle (MIP) detection was obtained up to now, but tests on the Macropad circuit (which integrates very low noise electronic readout of about $30 \mathrm{e}^{-} \mathrm{rms}$ ) were only performed with sensors with a maximum depleted thickness of about 15 $\mu \mathrm{m}$. Nevertheless, detection of $5.9 \mathrm{keV} \mathrm{X-rays} \mathrm{was} \mathrm{demon-}$ strated on such detectors and efficient detection of few $\mathrm{keV}$ electrons was also shown. Finally, measurements performed on $6.6 \mu \mathrm{m}$ wide strips separated by $4 \mu \mathrm{m}$ have shown an interesting potential of the TFA technology for the construction of very high spatial resolution detector, as low charge sharing was suggested by the detection of 10 to $50 \mathrm{keV}$ electrons. The overall results are promising regarding the use of TFA technology for some applications in radiation detection, though several problems need to be addressed if this is to become a viable solution for particle detection in high energy physics.

\section{ACKNOWLEDGMENT}

The authors would like to thank the following colleagues and collaborators for their important contribution and help in the work presented: C. Ballif, C. Miazza, and S. Dunand for the discussions, depositions, and optimizations of the a-Si:H sensors; W. Riegler for very useful discussions on the signal induction; D. Campos and M. Fransen for their help in the measurements of the aSiHtest detectors; and J. Morse and I. Snigireva for the EBIC measurements performed at the ESRF in Grenoble.

\section{REFERENCES}

[1] N. Wermes, "Pixel detectors for tracking and their spin-off in imaging applications," Nucl. Instrum. Methods Phys. Res. A, vol. A541, pp. $150-165,2005$.

[2] J. E. Brau, "The science and challenges for future detector development in high-energy physics," presented at the SNIC Symp., Stanford, CA, 2006

[3] M. Moll et al., "Development of radiation tolerant semiconductor detectors for the super-LHC," Nucl. Instrum. Methods Phys. Res. A, vol. A546, pp. 99-107, 2005.

[4] P. Jarron et al., "A new concept of monolithic silicon pixel detectors : Hasydrogenated amorphous silicon on ASIC," Nucl. Instrum. Methods Phys. Res. A, vol. A518, pp. 366-372, 2004.

[5] M. Despeisse, "Characterization of a hydrogenated amorphous silicon sensor deposited on integrated circuit for particle detection," $\mathrm{Ph}$.D. dissertation, Institut National des Sciences Appliquées de Lyon, Lyon, France, 2006.

[6] N. Kishimoto et al., "Radiation resistance of amorphous silicon in optoelectric properties under proton bombardment," J. Nucl. Mater., vol. 258-263, pp. 1908-1913, 1998.

[7] J. R. Srour et al., "Damage mechanisms in radiation-tolerant amorphous silicon solar cells," IEEE Trans. Nucl. Sci., vol. 45, no. 6, pp. 2624-2630, Dec. 1998.

[8] V. P. Mendez et al., "Hydrogenated amorphous silicon pixel detectors for minimum ionizing particles," Nucl. Instrum. Methods Phys. Res. A, vol. A273, p. 127, 1988.

[9] A. Kolodziej, "Staebler-Wronski effect in amorphous silicon and its alloys," Opto-Electr. Rev., vol. 12, no. 1, pp. 21-32, 2004. 
[10] N. Wyrsch et al., "Development of vertically integrated imaging and particle sensor," in Proc. Materials Research Society Spring Meeting, San Francisco, CA, Apr. 2003, vol. 762, pp. 205-210.

[11] J. Dubeau et al., "Response of amorphous silicon p-i-n detectors to ionizing particles," Nucl. Instrum. Methods Phys. Res. B, vol. B54, pp. 458-471, 1991.

[12] C. Hordequin et al., "Nuclear radiation detectors using thick amorphous-silicon MIS devices," Nucl. Instrum. Methods Phys. Res. A, vol. A456, pp. 284-289, 2001.

[13] G. Anelli et al., "A high-speed low-noise transimpedance amplifier in a $0.25 \mu \mathrm{m}$ CMOS technology," Nucl. Instrum. Methods Phys. Res. A, vol. A512, p. 117, 2003.

[14] M. Despeisse et al., "Characterization of $13 \mu \mathrm{m}$ and $30 \mu \mathrm{m}$ thick hydrogenated amorphous silicon diodes deposited over CMOS integrated circuits for particle detection applications," Nucl. Instrum. Methods Phys. Res. A, vol. A518, pp. 357-361, 2004.

[15] D. Moraes et al., "A novel low noise hydrogenated amorphous silicon pixel detector," J. Non-Cryst. Solids, vol. 338-340, pp. 729-731, 2004.

[16] F. Meillaud et al., "Efficiency limits for single-junction and tandem solar cells," Sol. Energy Mater. Sol. Cells, vol. 90, pp. 2952-2959, 2006.
[17] J. B. Chévrier and B. Equer, "High electric-field amorphous silicon p-i-n diodes: Effect of the p-layer thickness," J. Appl. Phys., vol. 76, no. 11, pp. 7415-7422, 1994.

[18] W. Riegler, "Extended theorems for signal induction in particle detectors VCI 2004," Nucl. Instrum. Methods Phys. Res. A, vol. A535, pp. 287-293, 2004.

[19] S. Qureshi et al., "Signal generation in a hydrogenerated amorphous silicon detector," IEEE Trans. Nucl. Sci., vol. 36, no. 1, pp. 194-198, Feb. 1989.

[20] Q. Gu et al., "High field electron-drift measurements and the mobility edge in hydrogenated amorphous silicon," Phys. Rev. B, vol. 52, pp. 5695-5707, 1995.

[21] Q. Gu et al., "Non-Gaussian transport measurements and the Einstein relation in amorphous silicon," Phys. Rev. Lett., vol. 76, pp. 3196-3199, 1996.

[22] J. D. Eskin, H. H. Barrett, and H. B. Barber, "Signals induced in semiconductor gamma-ray imaging detectors," J. Appl. Phys., vol. 85, pp. 647-659, 1999. 